\title{
SOBRE LA EXISTENCIA DE UNA NOVELA POLICÍACA ESPAÑOLA: UN BREVE REPASO A SUS PRINCIPALES TÍTULOS, AUTORES E HITOS
}

\author{
Diego Ernesto Parra Sánchez
}

\author{
Universidad del País Vasco
}

\begin{abstract}
Resumen: Este artículo pretende reflexionar sobre la conveniencia o inconveniencia de hablar de novela detectivesca española como una sólida e influyente tendencia policíaca a semejanza de otras como la británica, la francesa o la estadounidense. Con este objetivo, además de echar un vistazo a los principales argumentos tanto a favor como en contra de dicha consideración, se ahondará tanto en los títulos y en sus autores más representativos, como en los acontecimientos políticos, sociales y económicos que la han rodeado desde su origen.
\end{abstract}

Palabras clave: tendencia, detectivesca, hito, española, debate

\begin{abstract}
This article intends to reflect on the convenience or inconvenience of talking about Spanish detective novel as a solid and influential crime novel trend as the British, the French or the U. S. crime fiction trends. With this aim, apart from having a look to the main arguments either in favor or against this consideration, this work delves into the most important titles and the most representative authors, on the one hand, and into the political, social and economical events which have surrounded the Spanish crime fiction from its origin.
\end{abstract}

Key words: trend, detective, event, Spanish, discussion

\section{Introducción}

A la hora de hablar de tradición literaria policial en España o, siquiera, de novela policíaca española lo primero que cabe decir es si de verdad se puede hablar del policíaco español como una tendencia criminal lo suficientemente sólida e influyente como para ser tratada de manera individualizada, es decir, como una corriente literaria con unas marcas identitarias lo suficientemente arraigadas, unos rasgos compositivos singulares y una producción editorial tan amplia como para poder hablar de tradición policíaca. No son pocos los autores y críticos que se han debatido sobre este punto a lo largo de las últimas décadas, argumentando tanto a favor como en contra de la conveniencia de abordarla como tal en base a criterios tanto literarios -el número de autores dedicados exclusivamente a la misma, su prestigio, sus suficientes o insuficientes peculiaridades significativas o su empuje editorial- como extraliterarios: 
Sobre la existencia de una novela policíaca española:

un breve repaso a sus principales títulos, autores e hitos

por ejemplo los condicionamientos sociales, políticos y económicos que han orbitado en torno a este tipo de discursos en España y que han podido condicionar su desarrollo.

A lo largo de este artículo, se va a presentar una visión panorámica sobre la historia de este discurso narrativo y su práctica en este país aludiendo, en primer lugar a la polémica terminológica sobre la conveniencia o no de considerar esta práctica escritural como una tendencia literaria sólida, a la manera de la británica, la estadounidense o la francesa y, en segundo, revisando los hitos fundamentales que han jalonado su desarrollo desde su origen al boom editorial posterior a la Transición democrática.

Con respecto al primer punto, se presentará una recopilación crítica de las principales posturas, tanto a favor como en contra de dicha ponderación, haciendo especial hincapié en los argumentos esgrimidos por investigadores y estudiosos especializados en este género a lo largo de las últimas décadas. Y en lo relativo al segundo punto, este trabajo pretende ofrecer, del mismo modo, un registro de algunos de los autores, las obras y, sobre todo, las señas contextuales que, de una manera u otra, han condicionado el periplo vital de esta modalidad discursiva entre nuestras letras: desde su escasa y poco relevante producción -salvando un par de casos- durante el siglo XIX y gran parte del XX, a la ya mencionada eclosión durante la segunda mitad de la década de los setenta.

\section{La novela policíaca española como tendencia literaria: argumentos a favor y en contra}

Es un hecho incuestionable que la literatura policíaca ha estado condenada al ostracismo a nivel académico durante muchos años. Debido, por una parte, a su errónea consideración como "subliteratura" o "literatura de masas" y, por otra, a la incapacidad contumaz de las élites académicas de reconocer el axioma, como señala José Francisco Colmeiro en La novela policíaca española: teoría e historia crítica -una obra señera en el campo de la investigación sobre no solo el policíaco español, sino sobre el género a nivel global- de que "la realidad del hecho literario es mucho más compleja que la división entre literatura de kiosko y literatura de librería y no admite fácilmente estas simplificaciones."

Sin embargo, en parte acompañada por la extraordinaria buena salud de la que goza el género en la actualidad, tanto a nivel de calidad de autores como de promoción editorial, la crítica y la investigación literaria han vuelto los ojos hacia esta modalidad y cada vez son mayores los autores, los congresos y las publicaciones teóricas nacidas a partir del género y sus principales polémicas. Por lo que respecta a este artículo, una de las controversias que más debate ha suscitado y suscita todavía en la actualidad es el de

${ }^{1}$ José Francisco COLMEIRO, La novela policiaca española: teoría e historia crítica, Barcelona, Anthropos, 1994, 22. 
si la literatura de este género que se ha practicado en nuestro país desde su origen merece la acuñación del término "tendencia policíaca española".

Entre los autores que están a favor se encuentra el profesor Vallés Calatrava. En su libro La novela criminal española, por ejemplo, sostiene que sí existen suficientes rasgos diferenciadores de tendencia y señala el encuadramiento de la acción dentro del panorama social de este país y la utilización recurrente de personajes arquetípicos del imaginario español en las tramas para apoyar esta visión: “Todos estos componentes narrativos aparecen incluidos y determinados por la realidad española en todas sus vertientes y a ella se alude frecuentemente en las obras literarias de esta clase por servir de marco espaciotemporal donde se desarrolla la acción y actuar, además, como un elemento de representación social."’2

Por su parte, en el artículo "La novela negra en España", el estudioso del género Juan Carlos Martini hace hincapié en la variedad lingüística como peculiaridad diferencial del policíaco español como tendencia por sí misma: "resulta inevitable observar que [...] el tema de la literatura policiaca en España abarca problemas culturales, sociales y políticos entre los cuales debe situarse a un tiempo la diversidad de lenguas coexistentes."3 A diferencia de otras modalidades, esta sería la única que contaría con más de un idioma -las lenguas vernáculas que conforman el panorama lingüístico español- como vehículo expresivo, siendo el castellano y el catalán las más privilegiadas al estar sus autores arraigados en zonas donde existe una mayor industrialización y un desarrollo urbano más elevado: principalmente Madrid y Barcelona. Además, el crítico y ensayista argentino parece aludir al hecho de que esta misma diversidad idiomática constituye, a su vez, un ingrediente singular más de la problemática político-social que se desprende de este policíaco.

Otras dos voces acreditadas que defienden esta postura son la profesora Bados Ciria y el hispanista francés Yvan Lissorgues. En su artículo "La novela policiaca española y el canon occidental", la primera presenta un análisis del policíaco practicado en España, incidiendo en los autores más actuales cuyas publicaciones datan como mínimo de 1990 en adelante, con especial atención a la narrativa de Lorenzo Silva y a su saga protagonizada por la pareja de guardias civiles Bevilacqua y Chamorro, o a José María Guelbenzu y Alicia Giménez Bartlett con sus respectivas series protagonizadas por la juez Mariana de Marco y la inspectora Petra Delicado respectivamente. En síntesis, tras repasar la historia del género practicado en nuestro país desde sus orígenes, concluye que este, si bien coincide en algunos rasgos con el practicado por los maestros estadounidenses, se debe diferenciar nítidamente del policíaco practicado en el resto de Europa durante la primera mitad de siglo gracias a aspectos como las alusiones intertextuales o la introducción de reflexiones metaficcionales: "en la novela policíaca española hay una preponderancia de la semiótica detectivesca organizada según una

2 José Rafael VALLÉS CALATRAVA, La novela criminal española, Granada, Ediciones Universidad de Granada, 1991, 87.

3 Juan Carlos MARTINI, "La novela negra en España”, in: El procedimiento, Barcelona, 1980, 7. 
Sobre la existencia de una novela policíaca española:

un breve repaso a sus principales títulos, autores e hitos

orientación mimética del arte, aunque mediatizada por componentes ficcionales divergentes ajenos a tal tipo de novela, entre ellos, destacan el análisis político, la consideración filosófica general, la reflexión personalizada, y la intertextualidad, [...] también, la inscripción de discursos metaficcionales. De manera que [...] el género detectivesco español diverge en muchos aspectos del canon europeo de la primera mitad del siglo XX, pero converge, con algunos matices, con el modelo estadounidense que surge a partir de los años treinta. ${ }^{4}$

De la misma manera, el autor galo se pronuncia a favor de esta apreciación en su trabajo "La novela detectivesca española actual: un posibilismo Realista". En ella, destaca también la capacidad metatextual y el compromiso político como dos rasgos de una relevancia más que notable en las propuestas literarias de estos autores, junto con una "tendencia a tender puentes con otros textos, bien dentro del género policíaco, bien fuera de él"5 lo bastante recurrente y singular como para ser considerada una característica específica.

Sin embargo, la falta de una hornada consolidada de autores y obras de género criminal español precedente a su eclosión editorial en los años setenta, así como la aplicación reiterativa de la fórmula hard boiled como piedra angular sobre la que se erigen propuestas dotadas de escasos rasgos distintivos -apenas ciertos matices autóctonos-, son algunos de los argumentos esgrimidos a favor de cuestionar la conveniencia de hablar de policiaco español. Como sostiene el autor cubano Alejo Carpentier en "Papel del novelista", uno de los ensayos que componen La novela latinoamericana en vísperas de un nuevo siglo y otros ensayos (1981): "Una tradición de la novela existe, no lo olvidemos, cuando hay un movimiento de la novela, una escuela de la novela, una evolución de la novela"; así, señala la novela picaresca como ejemplo de relato novelesco del que sin lugar a dudas podemos hablar en términos de tradición en España. ${ }^{6}$ No así en el caso de la novela policíaca.

Uno de los primeros críticos en cuestionarse sobre este tema fue Juan del Arco, quien en 1948 publica un artículo en el extinto periódico El Español-recogido bajo el muy significativo título "El detective no puede llamarse Fernández"- en el que, pasando revista a la exigua nómina de obras y autores dedicados al género en España, daba ya testimonio adelantado sobre la falta de tradición policíaca en España aduciendo razones que, aunque de carácter eminentemente etnicistas, no dejan de suponer un primer peldaño interesante sobre este tema con tesis como "la particular naturaleza latina proclive a anteponer lo visceral frente al racionalismo o el cientificismo

\footnotetext{
${ }^{4}$ María Concepción BADOS, "La novela policíaca española y el canon occidental", in: 1616. Revista de la sociedad española de Literatura General y Comparada, vol. XI, Madrid, 2006, 145.

${ }^{5}$ Yvan LISSORGUES, "La novela detectivesca española actual: un posibilismo Realista", in: Juan VILLEGAS (ed.) Actas del XI Congreso de la Asociación Internacional de Hispanistas, Tomus I, Irvine, 1992, 176.

${ }^{6}$ Alejo CARPENTIER, La novela latinoamericana en vísperas de un nuevo siglo y otros ensayos, México D.F., Siglo Veintiuno, 1981, 36.
} 
anglosajón o la predilección del lector por ambientes exóticos y escapistas alejados de la realidad a pie de calle." 7

Ya en épocas más recientes, el pormenorizado estudio monográfico sobre el perfil del investigador en el policíaco español The Spanish Sleuth: the detective in Spanish Fiction, de la profesora estadounidense Patricia Hart, supone un trabajo de referencia que sigue este posicionamiento argumental. En el mismo, tras hacer mención del conjunto de escritores que empiezan a alcanzar notoriedad tras el fin de la dictadura y la transición democrática como la única generación sobresaliente del género en España -con el denominador común en todos ellos de utilizar este formato para introducir un retrato realista crítico del paisaje urbano de la España de esa época-, establece una conexión clara entre estos y los autores estadounidenses de los años treinta y cuarenta, en especial Hammett y Chandler, aseverando, en este sentido, que la influencia de la novela negra estadounidense sobre estos autores españoles es tan manifiesta, que llega incluso "hasta el punto de no invocar nada realmente novedoso o distintivo." 8 Otros autores norteamericanos, como el profesor William J. Nichols, se adhieren también a esta argumentación. En otro monográfico, en este caso titulado Transatlantic mysteries: crime, culture and capital in the "noir novels" of Paco Ignacio Taibo II and Manuel Várquez. Montalbán, encuadrado dentro de los estudios transatlánticos y sobre la obra de estos dos autores, este autor indica que "La ficción noir, también referida como tradición norteamericana hard boiled, ofrece a ambos autores el vehículo perfecto para esa finalidad [la de criticar el sistema neoliberal instaurado en occidente] a través de un género de ficción popular irónicamente localizado donde el atractivo de mercado y la literatura se cruzan." 9

El propio Manuel Vázquez Montalbán, autor referente de esta generación, tampoco es ajeno a esta controversia. Apunta que, aparte de que no existen suficientes características diferenciales como para poder hablar de una novela policiaca española de hecho, en su libro La literatura en la construcción de la ciudad democrática afirma en este sentido que "Hoy día casi no existe ninguna cultura nacional-patriótica y las literaturas nacionales solo lo son por la lengua y ya muy poco por la propia tradición literaria"10-, sostiene que esta consideración parece estar más apoyada en intereses de editoriales y de una buena parte de la crítica especializada que en criterios literarios: "Es evidente que todas las apariencias conspiran para decir que sí, que existe una novela negra en España. Por ejemplo, surgen colecciones dedicadas a la novela policial. La crítica habla $[\ldots]$, con paternalismo, [...] sobre la existencia de un "boom". Incluso se resalta el

\footnotetext{
7 Juan DEL ARCO, "El detective no puede llamarse Fernández", in: El Español, Madrid, 18 de septiembre de 1948 .

8 Patricia HURT, The Spanish Sleuth: The Detective in Spanish Fiction, Rutherford, Fairleigh Dickinson University Press, 1987, 34.

9 William J. NICHOLS, Transatlantic mysteries: crime, culture and capital in the "noir novels" of Paco Ignacio Taibo II and Manuel Várquez. Montalbán, Plymouth, Bucknell University Press, 2011, 11-12. 10 Manuel VÁZQUEZ MONTALBÁN, La literatura en la construcción de la ciudad democrática, Barcelona, Mondadori, 2001, 73.
} 
Sobre la existencia de una novela policíaca española:

un breve repaso a sus principales títulos, autores e hitos

hecho de que se está haciendo una campaña de difusión editorial de la novela negra, corrompiendo el gusto de los escritores, para llevarles hacia ese terreno por afán comercial. Todo eso deja un clima, un saber convencional en torno a que sí, a que existe, a que hay unos cultivadores. Saberes convencionales que pocas veces se plantean para ver hasta qué punto responden a la realidad."11

En cuanto a la falta de una tradición consistente de literatura policíaca española, se trata, sin duda, del argumento que más peso tiene y que con más recurrencia se cita entre los trabajos de autores y estudiosos del género. Siguiendo con las aportaciones negacionistas del creador de la saga Carvalho, este llega a concluir que no cabría lugar hablar de novela policiaca española debido a la carencia de una tradición culta dedicada al género y basada en un amplio acervo de obras y autores que lo hayan practicado desde su origen ofreciendo aportaciones novedosas y distintivas; más bien, en el caso de España, lo que se ha experimentado es una serie de acercamientos de un conjunto de novelistas a esta modalidad, habiéndose intentado hacer pasar toda una verdadera y palpable tradición añeja de literatura de consumo - "novelas de quiosco", colecciones de carácter folletinesco, etc.- por tradición literaria policíaca erróneamente. ${ }^{12}$ A esto mismo se refiere, no sin ironía, el profesor Juan Ignacio Ferreras cuando sostiene que "el día que se escriba la historia de la novela policíaca en España habrá que especificar por qué se han producido obras tan escasas en número y tan regulares en calidad"13, a lo que añade el profesor Juan Paredes Núñez que, si bien es cierto que se han publicado traducciones de los más importantes autores policíacos desde la década de los años treinta, con escritores como Poe o Collins a Chandler o Mac Donald pasando por Simenon entre los elegidos, nunca ha existido una tradición oriunda de este tipo de literatura y sería desacertado incorporar esta práctica editorial como muestra de un supuesto cultivo de la literatura policíaca, tanto a nivel lector como escritural, en España. ${ }^{14}$

Por último, el antes aludido Jose Francisco Colmeiro, acudiendo a motivos de raíz socio-político en su artículo "De Pepe Carvalho al subcomandante Marcos: la novela policíaca hispánica y la globalización” sostiene que habría otras razones de mayor peso que explicarían esta ausencia de una novela policíaca autóctona, "como la falta generalizada en el ámbito hispánico de estados de derecho apoyados en un orden jurídico burgués que garantice las libertades individuales, que es la base histórica e ideológica del género policíaco tradicional."15

11 Manuel VÁZQUEZ MONTALBÁN, "Sobre la inexistencia de la novela policiaca en España”, in: Juan PARADES NÚÑEZ (ed.), La novela policiaca española, Granada, 1989, 49.

12 Ibidem, 50.

${ }^{13}$ Juan Ignacio FERRERAS, Tendencias de la novela española actual, 1931-1968. Seguidas de un Catálogo de urgencia de novelas y novelistas españoles de la posguerra, París, Hispanoamericanas, 1970, 125.

14 Juan PAREDES, "El cuento policiaco en Pardo Bazán", in: Estudios sobre la Literatura y Arte dedicados al profesor Emilio Orozco Díaz, Tomus III, Granada, 1979, 7-18.

15 José Francisco COLMEIRO, "De Pepe Carvalho al subcomandante Marcos: la novela policíaca hispánica y la globalización", in: Revista Iberoamericana, vol. LXXVI, 2010, 478. 
Por todo ello parece más acertado hablar de práctica de literatura criminal autóctona - con ciertos rasgos en común y potenciada tanto cualitativa como comercialmente a partir de los setenta- frente al concepto de novela policíaca española. Pero, echando un vistazo desde la situación actual de esta práctica discursiva en España sobre su origen, su escaso desarrollo y su poco peso: ¿Cuáles han sido los factores que más determinantemente han condicionado este escaso, irregular e insustancial cultivo en nuestro país hasta su boom editorial?

\section{Breve repaso a la historia del género policíaco en España y sus condiciona- mientos contextuales}

Son tres las razones que, históricamente, se han enumerado en relación a la falta de una tradición consolidada de literatura policíaca española: razones políticas, razones de naturaleza estilística y razones sociohistóricas. En lo que atañe a las primeras, hay que comentar las complicaciones que podían representar su cultivo y promoción en un régimen autoritario como el franquista, sobre todo en su vertiente negra o hard boiled, por su dimensión realista y su inclinación inherente al retrato crudo de la realidad social a pie de calle. Esto explica que, durante el franquismo, tanto escritural como editorialmente, existiera una predilección por el relato clásico de enigma o whodunnit por sus ostensiblemente menores implicaciones críticas; las pocas muestras de novelas negras que llegaban, en cambio, eran pasto de la censura oficialista. Así ocurriría, como apunta Vallés Calatrava, con la publicación de Lady in the lake (1943) de Raymond Chandler que, publicada por la editorial Mateu ${ }^{16}$ en 1962 bajo el título La "dolce vita" en América, fue objeto de censura siendo varios pasajes mutilados ${ }^{17}$. Entre las razones de naturaleza estilística, sobresale la de "la consideración negativa que acompañó al género en nuestro país desde sus primeras manifestaciones y que obligaba a los pocos autores que practicaban el género a utilizar ubicaciones extranjeras para localizar sus tramas o a esconder sus identidades bajo pseudónimos ${ }^{18}$ ", como refiere el crítico José Fernández en su texto "Mitos imperfectos: Observaciones de un lector continental en torno a la novela policiaca". Efectivamente, las convenciones sociales y críticas de carácter elitista, que han sido las dominantes durante mucho tiempo en España, tendían a integrar la novelística criminal en el campo de la literatura de segunda fila generalizándose una infravaloración de la misma y que condicionó, sin duda, la exigua práctica de este tipo de literatura en este país. En palabras de Salvador Vázquez de Parga: "No ha habido en España escritores especializados en novela policiaca y dedicados a ella como su medio normal de expresión, [...] a causa de la deplorable consideración social [...] que ha

\footnotetext{
${ }^{16}$ Empresa editorial con sede en Barcelona fundada por Francisco F. Mateu en 1945 dedicada a la publicación de obras sensacionalistas, así como cómics o literatura infantil, tanto en castellano como en catalán.

${ }^{17}$ VALLES, op. cit., 80.

18 José FERNÁNDEZ, "Mitos imperfectos: Observaciones de un lector continental en torno a la novela policiaca", in: Revista de Occidente, Tomus V, Madrid, 1967, 10.
} 
Sobre la existencia de una novela policíaca española:

un breve repaso a sus principales títulos, autores e hitos

tenido en nuestro país." ${ }^{19}$ Sin embargo, ninguna de estas razones explica tan bien la ausencia de tradición literaria policíaca en España como ciertas motivaciones sociohistóricas. Como señala Colmeiro, este género "presupone unas condiciones particulares para su surgimiento tales como la existencia de un sistema democrático pluralista, una sociedad capitalista avanzada y una demografía urbana desarrollada."20 Por tanto, la novela policíaca surge en un contexto de legitimación de la ideología jurídica de la burguesía industrial, y es precisamente la falta de esta ideología la que explica, en última instancia, su inexistencia en España hasta la década de los setenta. Hay que esperar hasta el final de la dictadura franquista y el consiguiente advenimiento de la democracia para ver eclosionar esta literatura en un país donde se empiezan a dar los conflictos políticos, sociales y económicos propios de las ciudades modernas industrializadas bajo el amparo de una libertad de expresión y de edición que empieza a permitir la reflexión crítica -libre ya de las amputaciones propias de la censura dictatorial- sobre la realidad del momento y su problemática: principalmente las fisuras del movimiento transicional democrático provocadas por la subsistencia de agentes políticos continuistas entre las esferas gubernamentales. Sólo así se propicia la atmósfera que posibilita el desarrollo editorial del género en España como el surgimiento de un público lector cada vez más masivo: como sintetiza magistralmente la profesora Balibrea, "público e industria se retroalimentan mutuamente para generar un fenómeno cultural que surge durante la transición pero que no habría sido posible en su extensión y alcance sin los desarrollos socioeconómicos e ideológicos del tardofranquismo" 21 ; un contexto en el que el capitalismo, tardío y circunscrito a zonas concretas, aparece en su forma plena, y en los grandes centros urbanos surgen los fenómenos propios de la ciudad capitalista como la soledad, la violencia y la explotación.

Hasta este momento, su práctica se había limitado a un número reducido de autores y obras poco trascendentes. Su primera cultivadora stricto sensu, ya no solo como autora sino también a través de estudios críticos sobre el género, fue Emilia Pardo Bazán²2, quien publica en el año 1905 el relato "Misterio", y en 1911 "La cana" y "La gota de sangre". Caracterizados por un primitivismo tanto a nivel de estilo como a nivel argumental, se trata de textos que siguen fielmente el modelo británico de detective aficionado que resuelve un crimen por medio de la observación detallista y la deducción. Su contribución inaugural terminaría con la publicación en 1913 de Belcebú,

${ }^{19}$ Salvador VÁZQUEZ DE PARGA, La novela policiaca en España, Barcelona, Ronsel, 1993, 24.

${ }^{20}$ COLMEIRO, op. cit., 263.

${ }^{21}$ Mari Paz BALIBREA, "La novela negra en la transición española", in: Iberoamericana, Tomus II, Madrid, 2002, 113.

22 A diferencia de lo que una parte de la crítica especializada sostiene, en este trabajo no vamos a considerar a Pedro Antonio de Alarcón y su relato breve "El clavo" (1853) como autor y obra inauguradoras del género en España por entender que guarda mayor relación con el género de crónica de crímenes reales o causas célebres. 
aunque posteriormente el profesor Varela Jácome descubriría un manuscrito titulado Selva que podría suponer la continuación de La gota de sangre: un escrito inédito compuesto por ciento sesenta y ocho cuartillas redactadas a mano y llenas de tachaduras y correcciones, lo que, para Bados Ciria, parecería "demostrar el escaso interés de la autora por concluir esta novela y la pérdida de entusiasmo en cuanto a la literatura policial en sus últimos años."23 Anteriormente, en 1909, Joaquín Belda y Manuel A. Bedoga habían publicado ¿Quién disparó?: una novela parodia de Sherlock Holmes y el Dr. Watson que se hizo muy popular en la época entre unos lectores que empezaban a disfrutar con las traducciones de clásicos del género -principalmente exponentes del relato de enigma- anglosajones. En 1916, Enrique López y José Ignacio Alberti escribieron la obra de teatro titulada Sebastián el Bufanda o el robo de la calle Fortuny y, dos años más tarde, aparece la colección "La novela policíaca", que estaba dedicada exclusivamente a la publicación de obras de teatro de temática policíaca y compuesta por piezas escritas por autores españoles que seguían aplicando de manera estricta los convencionalismos del whodunnit, bien desde el pastiche, bien desde formulaciones paródicas. Tras el estallido de la guerra civil y la consiguiente llegada de la dictadura franquista, la publicación de esta literatura decae en gran medida y, aunque se siguen editando traducciones extranjeras, la censura lleva a cabo, como se ha apuntado, una labor de depuración más que notable en consonancia con los valores sociales que se pretendían fomentar. Es preciso destacar también que este valor cultural seguía considerándose entre el público español como un valor ajeno y extranjerizado. No solo el hecho de que la mayoría de las obras que se publicasen fueran traducciones de obras de otros países -o simples recreaciones emuladoras del relato de enigma-, sino también la recurrencia a investigadores foráneos y ubicaciones extranjeras ${ }^{24}$ era una constante en la época. Sin duda, el autor que mejor ha explicado este fenómeno -valiéndose de su vasta experiencia tanto como editor como escritor para la editorial Bruguera- es Francisco González Ledesma. En un artículo publicado 1987 bajo el título "La prehistoria de la novela negra" sintetizaba este fenómeno de la siguiente manera: "La gente de la calle no hubiera admitido inspectores Gómez ni criminales Rodríguez, ni calles conocidas que no excitaran sus sueños y sus ansias de viajar. Todo lo bueno sucedía entonces fuera de España, y las únicas policías con garantía de origen eran Scotland Yard y el FBI, sobre todo este último. [...] De modo que por esas razones tan importantes nadie escribía novelas policiacas ambientadas en las ciudades españolas y

\footnotetext{
${ }^{23}$ María Concepción BADOS, "Feminización de la novela policiaca: alternativas para el cambio sociocultural", in: Mercedes GONZÁLEZ DE SANDE (coord.), La imagen de la mujer y su proyección en la literatura, la sociedad y la bistoria, Tomus I, Sevilla, 2010, 31.

24 A la hora de caracterizar y localizar tanto personajes como ubicaciones, los escritores españoles se decantarán por dos países principalmente: Reino Unido y Estados Unidos. Los primeros fueron favorecidos por autores como Wenceslao Fernández Flores, Cèsar August Jordana o Luis Conde Vélez, mientras que Felipe Pérez Capo, Ángel Marsá Becal, Juan José Mira o Agustín Elías.
} 
Sobre la existencia de una novela policíaca española:

un breve repaso a sus principales títulos, autores e hitos

encima con sentido crítico, es decir lo que hoy llamaríamos ya "novela negra". Los argumentos se desarrollaban en Inglaterra, Estados Unidos y excepcionalmente en Francia. En lugares oficialmente tan corruptos era posible situar grandes "gangs", policías que cobraban bajo mano, gobernantes venales y hasta alguna señorita que enseñaba el portaligas, si bien esa prenda íntima nunca pudo mencionarse de una forma expresa." 25

El escritor Mario Lacruz y su novela El inocente (1953) suponen una interesante excepción en el panorama de escritores españoles de policíaco durante la posguerra. La novela de Lacruz escapa conscientemente de la tradición importada de novela policíaca clásica e inicia al mismo tiempo un acercamiento del género hacia formas de mayor calado por medio de una imbricación novedosa de, por un lado, el aspecto lúdico del interrogante policíaco ágilmente narrado, con, por otro lado, la observación profunda de lo humano y social y la experimentación técnica. Como indica el investigador Julián Luzán en su artículo de 1981 "Interrogatorio", el propio Lacruz reconoce en este sentido la influencia del autor belga Simenon, quien, como se ha referido en el tercer apartado de este capítulo dedicado al polar, incorpora la introspección psicológica enriqueciendo en gran medida esta literatura: "seguro que hay influencias de todas mis lecturas. De Simenon también [...] en la capacidad de, con muy pocos medios, crear un clima o un personaje y meterte enseguida en él."'26 Por su parte, para Colmeiro Lacruz combina elementos de vanguardia narrativa, como la profundidad psicológica, el perspectivismo o la ruptura del orden lógico temporal, con ingredientes clásicos de la intriga policiaca, obteniendo un resultado novedoso. ${ }^{27}$

En las décadas de los años cincuenta y sesenta, aparece los investigadores Manuel González, alias Plinio, de la policía municipal de Tomelloso, y su inseparable Don Lotario, el veterinario del pueblo. Con novelas como El reinado de Witiza (1967) o Las bermana coloradas (1969), la práctica del género en España le debe a Francisco García Pavón la creación de historias policíacas auténticamente enraizadas en nuestro país inaugurando una tendencia autóctona alejada de los moldes imperantes anglosajones, en la que enigma va cediendo terreno a favor del retrato costumbrista de La Mancha profunda, trasunto de la España rural bajo el régimen franquista, con una minuciosa descripción de lugares, costumbres y tipos humanos del momento.

Tras décadas de contribuciones escasas en número y limitadas en repercusión, salvando las aportaciones de García Pavón, Lacruz o la labor precursora de Pardo Bazán, el género experimenta el ya mencionado despegue literario en España en la década de los setenta. Impulsada tanto crítica como editorialmente, con la introducción masiva de traducciones, en este caso, de clásicos estadounidenses publicados por sellos

${ }^{25}$ Francisco GONZÁLEZ, "La prehistoria de la novela negra", in: Los cuadernos del norte, Tomus VIII, Oviedo 1987, 12.

${ }^{26}$ Julia LUZÁN, "Interrogatorio", in: Gimlet: revista policíaca y de misterio, Tomus IV, Barcelona $1981,36$.

${ }^{27}$ COLMEIRO, op. cit., 141.

60 | Acta Hispanica, Hungría 20: 51-62, 2015, ISSN: 1416-7263 
como Enlace o Bruguera, florece definitivamente la novela policíaca en nuestro país tanto en castellano como en catalán. Así, con De mica en mica s'omple la pica (publicada en castellano como El procedimiento) de Jaume Fuster y Tatuaje de Manuel Vázquez Montalbán -publicadas en 1972 y 1974 respectivamente- comienza la etapa de mayor y mejor producción de narrativa policíaca de nuestro país con la fórmula de la novela negra estadounidense como referencia y propiciada por los condicionamientos sociales, económicos y políticos anteriormente explicados, sin los cuales esta generación de escritores no hubiera emergido nunca. De entre todos, destaca la figura del autor de la serie Carvalho, no sólo por su labor precursora o la fama internacional alcanzada ${ }^{28}$, sino por la riqueza literaria de sus propuestas. La apropiación y la asimilación de la ficción detectivesca hard-boiled de Montalbán debe ser entendida como un medio para contemplar e interpretar la realidad en dos direcciones principales: en primer lugar, como una crónica de la Transición española y, en segundo, como un vehículo expresivo al servicio de la canalización del desencanto consecuencia de la frustración generada tras constatar la ausencia de cambios o transformaciones nucleares más allá de una aparente revolución impostada y vacía de contenido. Tras empezar con Yo maté a Kennedy en 1972, la saga alcanza una gran popularidad a finales de los setenta con la publicación de la Soledad del manager (1977) y Los mares del sur (1979) en la que analiza con detalle el nacimiento y la infiltración de un capitalismo de consumo mientras se revelan las grietas de todo un sistema económico sin regular y objeto de especulación apoyada por una serie de medidas económicas impulsadas por los distintos gobiernos postransicionales y que se consolidan en la década de los noventa. Son los entresijos de esta nueva realidad española y la problemática que suscita a nivel social (con aspectos como desigualdad, la corrupción o la inseguridad marcando el día a día a pie de calle) los que aborda esta novelística en la que, argumentalmente, la investigación persigue, por encima del esclarecimiento del enigma, no la restauración de un orden quimérico, sino el descubrimiento del desequilibrio subyacente y sus agentes inductores que penetra en todas las esferas de la sociedad.

Con Montalbán, descollarían un raudal de autores (Jaume Rivera, Juan Madrid, Julián Ibáñez, Gónzalez Ledesma, Martínez Reverte, Eduardo Mendoza o Andreu Martín) que seguirán publicando hasta bien entrados los noventa -junto con la irrupción de una segunda hornada de autores entre los que destacan Eugenio Fuentes o Lorenzo Silva- con propuestas en las que, si bien se pueden reconocer características comunes como la crítica social -a menudo con la intercesión del humor como elemento distanciador-, la recreación costumbrista o la acumulación de múltiples referencias metaficcionales sobre el propio género, no se podría hablar de novela policíaca española en términos de tendencia o corriente literaria per se.

\footnotetext{
${ }^{28}$ Véase la influencia ejercida sobre otros escritores europeos de género policíaco posteriores como, por ejemplo, el italiano Andrea Camileri, cuyo investigador central recibe el nombre de Comisario Montalbano en claro homenaje a la figura del escritor barcelonés.
} 
Sobre la existencia de una novela policíaca española:

un breve repaso a sus principales títulos, autores e hitos

\section{Conclusión}

A la vista de lo referido, se puede concluir que, si bien es necesario no solo un número destacado de autores que practiquen el género (con el consiguiente empuje editorial), sino los condicionantes históricos propicios (tanto desde el punto de vista político, como desde el social y el económico) para que pueda irrumpir una narrativa criminal novedosa y de marcados rasgos diferenciadores pudiéndose, por tanto, hablar de tendencia policíaca, en el caso de la literatura detectivesca practicada en España, solo podemos hablar de práctica literaria autóctona con ciertos rasgos en común pero basada en la repetición reiterativa de los patrones del relato de enigma o whodunnit británico, hasta la década de los setenta, y de la novela negra o hard boiled de los maestros estadounidenses a partir de esa fecha. Y esto debido a factores tanto literarios, como el reducido número de autores dedicados al género, dada, entre otras cosas, la baja consideración de esta literatura o su escaso impulso editorial en nuestro país, como motivos ajenos a los parámetros de la creación literaria, como la censura franquista o la ausencia de una revolución industrial profunda que cambiara de raíz el contexto económico, social y político de España propiciando, de esta modo, los conflictos propios de las sociedades urbanas desarrolladas en las que este discurso arraiga: como la lucha de clases, la injusticia, la desigualdad o la violencia que, en consecuencia, estas van dejando a su paso. 